\title{
Prevención de la transmisión vertical y efectos secundarios de la terapia anti-retroviral en hijos nacidos de madres seropositivas para VIH en el área Sur-Oriente de Santiago, Chile: perspectiva de 15 años
}

\author{
Anamaría Peña, Inés Cerón, Isolda Budnik, Diego Barttlet, Karina Cataldo, \\ Ana M. Fernández, Anamaría Ramírez y Martín Lasso
}

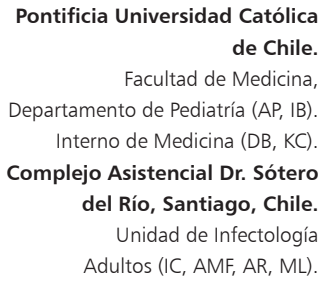

Pontificia Universidad Católica de Chile.

Facultad de Medicina Departamento de Pediatría (AP, IB) Interno de Medicina (DB, KC). Complejo Asistencial Dr. Sótero del Río, Santiago, Chile. Unidad de Infectología Adultos (IC, AMF, AR, ML)

Lugar donde se efectuó el estudio: Complejo Asistencial Dr. Sótero del Río.

Conflictos de interés: Los autores no declaran conflictos de interés Financiamiento: No hubo financiamiento.

Recibido: 21 de enero de 2013 Aceptado: 10 de octubre de 2013

Correspondencia a: Anamaría Peña Donat anapena@med.puc.c Cotapos 1091, Independencia. Fonos: 56-2-25764626 / $56-2-27349542$ Fax: 56-2-5764625

\section{Introducción}

E n la última década, la epidemia de la infección por VIH ha mostrado un aumento en la proporción de mujeres que viven con el VIH. En América Latina un tercio de los infectados son mujeres ${ }^{1}$. En Chile también se ha presentado esa tendencia, con una disminución de la razón hombre/mujer desde 6,6 en el quinquenio 19861990 a 3,8 entre 2006-2010². Este hecho es importante, considerando que la principal vía de transmisión del virus en los niños es la vertical. Según datos sudamericanos en el 2011, la tasa de infección en mujeres embarazadas oscilaba entre $0,03 \%$ (Nicaragua) y $0,8 \%$ (República Dominicana) y la de transmisión vertical (TV) entre $0,5 \%$ (Panamá) y 31\% (Guatemala). Independientemente de la cifra, esta vía de infección es responsable de $92 \%$ de las infecciones en niños ${ }^{3}$.

A nivel nacional, la prevención de la TV se aplica desde el año 1997, inicialmente como monoterapia con zidovudina tanto a la madre como al recién nacido y posteriormente con bi y triterapia, según los nuevos protocolos, lo que ha permitido que la tasa de TV en binomios madre-hijo controlados disminuyera de $30 \%$ hasta 1996 a $1,6 \%$ en el año $2010^{2,4,5}$.

A pesar del evidente beneficio de la terapia anti-retroviral (TARV), ésta no está exenta de efectos adversos tanto en los recién nacidos como en las mujeres embarazadas. En los recién nacidos los más frecuentemente mencionados son las alteraciones hematológicas (citopenias), que en la mayoría de los casos son leves o no tienen repercusiones clínicas graves ${ }^{6-8}$; toxicidad mitocondrial ${ }^{9}$, convulsiones febriles precoces ${ }^{10}$, entre otros. Respecto a patología obstétrica, se ha descrito un mayor riesgo de pre eclampsia y muerte fetal con el uso de TARV de alta eficacia (HAART) previa al embarazo ${ }^{11}$, así como mayor riesgo de parto prematuro y diabetes gestacional con el uso de inhibidores de proteasas (IP) o de acidosis láctica asociada al uso de didanosina y estavudina ${ }^{12}$. Después de 
más de dos décadas de uso de TARV orientada a la prevención de la infección del hijo de madre seropositiva, no se cuenta con datos nacionales que describan los efectos adversos en los recién nacidos, por lo que la realización de este estudio apunta a aportar dicha información.

El objetivo de este estudio es determinar la tasa de $\mathrm{TV}$, frecuencia y evolución de los efectos adversos posiblemente derivados de la TARV en los hijos de madres seropositivas para VIH.

\section{Pacientes y Métodos}

\section{Diseño}

Estudio observacional, descriptivo, que informa los resultados encontrados en forma retrospectiva del seguimiento y estudio clínico rutinario bajo normativa local de un grupo de binomios madre VIH-positiva/hijo controlados en el Complejo Asistencial Dr. Sótero del Río (CADSR), dentro del Programa VIH/SIDA del SSMSO, entre enero de 1995 y junio de 2010.

\section{Recolección de datos}

Variables demográficas y clínicas, tanto de las madres como de los hijos, fueron recolectadas de las fichas clínicas respectivas y registradas en una base de datos prediseñada. Estas variables incluyeron: En las madres: datos demográficos; etapa clínica y enfermedades marcadoras; recuento y porcentaje de linfocitos T CD4 basales y carga viral al diagnóstico de la infección por $\mathrm{VIH}$, durante el embarazo y al parto; temporalidad diagnóstica de infección por VIH respecto al embarazo; uso de TARV previa y durante el embarazo; co-morbilidades en la gestación y estatus serológicos para virus hepatitis B (VHB), virus hepatitis C (VHC), Toxoplasma gondii, enfermedad de Chagas y sífilis; En los hijos: edad gestacional; vía de parto; peso al nacer y su relación con la edad gestacional; puntaje de Apgar; patología fetal; recuento y porcentaje de linfocitos T CD4 al nacer; resultados de reacción de polimerasa en cadena (RPC) para VIH seriados de acuerdo al protocolo nacional de diagnóstico realizados por el Instituto de Salud Pública (ISP) ${ }^{13}$; TARV; lactancia materna; co-morbilidades y efectos colaterales de la TARV.

\section{Protocolo de prevención de transmisión vertical (PPTV)}

Previo al año 2005, este protocolo consideraba tratamiento para las madres con monoterapia con zidovudina o biterapia con zidovudina y lamivudina desde el momento del diagnóstico del embarazo. Excepcionalmente algunas madres recibían triterapia con nevirapina o algún IP. A partir del año 2005 y coincidente con la incorporación como patología $\mathrm{GES}^{14}$, el protocolo considera uso de triterapia con IPs desde la semana 24 de gestación, si la madre no tiene indicación per se de TARV. Por su parte, todas las madres reciben una infusión de zidoduvina ev $4 \mathrm{~h}$ previas a la cesárea. En el caso que la madre no haya recibido TARV durante el embarazo además se administra nevirapina intraparto. Posteriormente, los recién nacidos reciben zidoduvina por seis semanas, asociada a nevirapina cuando ésta se usó como profilaxis durante el embarazo o en el parto, y a otros anti-retrovirales cuando la madre tiene resistencia conocida a anti-retrovirales ${ }^{15}$.

\section{Seguimiento de los recién nacidos}

Se realiza de manera semanal durante el período de ingesta de la profilaxis y luego mensual hasta los seis meses de edad, en la Unidad de Infectología Pediátrica. En cada visita se determina antropometría, examen físico y exámenes de laboratorio de acuerdo a criterio del médico tratante y anormalidades encontradas: hemograma, recuento de plaquetas, gases venosos, electrolitos plasmáticos, pruebas de función hepática y renal, CPK, ácido láctico, cultivo rápido (shell vial) para $\mathrm{CMV}$ durante las primeras dos semanas de vida y estudio de otras infecciones transplacentarias cuando no se dispone de las serologías maternas (T. gondii, VHB, VHC y Chagas). Los exámenes de laboratorio alterados se repiten hasta su normalización y el volumen de extracción de sangre no sobrepasa el máximo recomendado por la NIH Clinical Center Guidelines ${ }^{16}$. La extracción de sangre para ácido láctico se efectúa por punción de yugular externa, se deposita en tubo heparinizado previamente refrigerado, transportándose en hielo inmediatamente al laboratorio para ser procesado dentro de 20 min por técnica enzimática automatizada. Para el diagnóstico de acidosis láctica se efectúa cálculo del anión gap. Las toxicidades se clasificaron según una graduación pre-establecida para niños ${ }^{15}$.

\section{Análisis estadístico}

Los datos fueron analizados utilizando los programas MedCalc ${ }^{\circledR}$ y SSPS 17.0. Se utilizó descriptores estadísticos habituales (promedio, desviación estándar, mediana y rango). En el análisis univariado, Test de Chi cuadrado y exacto de Fisher fueron usados para el análisis de variables categóricas y el test $\mathrm{t}$ para comparar medias de variables continuas distribuidas de forma normal. Para el análisis multivariado se utilizó modelo de regresión de Cox. Variables con un valor $\mathrm{p}<0,1$ en el análisis univariado fueron incluidas en el modelo multivariado. Las diferencias se consideraron significativas para valores $\mathrm{p}<0,05$.

\section{Resultados}

Durante el período de estudio (1995 al 2010) se identificaron 101 binomios madre seropositiva/hijo observándose un significativo incremento en la mediana 


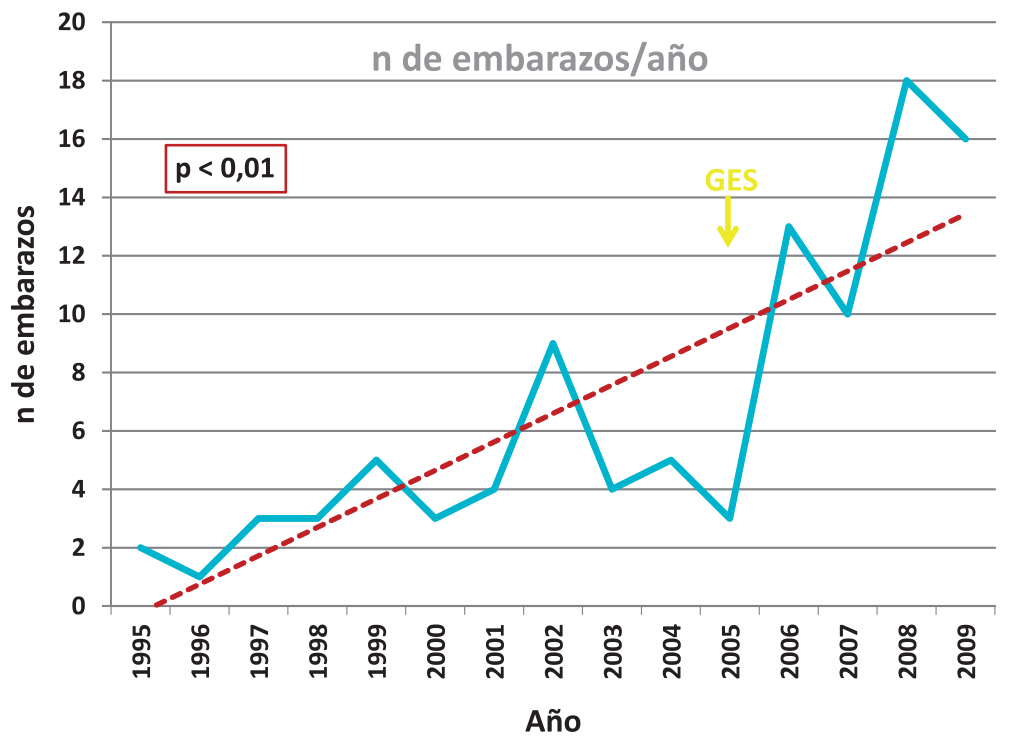

Figura 1. Distribución anual embarazos con protocolo de prevención de la transmisión vertical de VIH. Nota: En el gráfico no están considerados los embarazos del año 2010 pues correspondió sólo a medio año de seguimiento.

\section{Tabla 1. Resumen de las principales características clínicas de las mujeres embarazadas} con infección por VIH

Variables

n $(\%)$

Edad, años, promedio \pm DS

$27,4 \pm 6,3$

Co-morbilidades previas al embarazo

Patología psiquiátrica

$9(9,6)$

Patología gineco-obstétrica

$3(3,2)$

Hipertensión arterial

$2(2,1)$

Otros $^{8}$

$6(6,4)$

\section{Co-morbilidades durante el embarazo}

Anemia

Diabetes gestacional

Infección del tracto urinario

Síndrome hipertensivo del embarazo

Infecciones oportunistas

\section{Momento del diagnóstico de la infección por VIH}

Previo al embarazo

$47(50)$

Concomitante con el embarazo

$45(47,9)$

Intraparto

$(2,1)$

Estadío de la infección por VIH al momento del diagnóstico

Etapa de SIDA

Enfermedades marcadoras de SIDA

Neumonía por $P$. jiroveci

Candidiasis esofágica

Infección por micobacterias

Otras*

Recuento de linfocitos CD4 (x 103/. $\left.\mathrm{mm}^{3}\right)$ [Mediana (Rango)]

$\%$ de linfocitos CD4 [Mediana (Rango)]

NOTA: \&asma 2, sd. ovario poliquístico 1, epilepsia 1, displasia de cadera operada 1, psoriasis 1; * meningitis criptocócicca 1 , retinitis por CMV 1, herpes zoster diseminado 1 , carcinoma cérvico-uterino 1 , sarcoma de Kaposi 1.

anual de embarazos posterior al año 2005, coincidente con la incorporación del VIH/SIDA como patología GES, y que va de 3 (rango: 1-9) embarazos/año en el período 1995-2005 a 14,5 (rango: 10-18) embarazos/año a contar del año 2006 ( $\mathrm{p}<0,01)$ (Figura 1).

Se excluyeron siete binomios por fichas incompletas y el análisis se efectuó en 94 embarazos y 96 hijos (dos embarazos gemelares). Dentro de los 94 embarazos, siete correspondieron a un segundo embarazo de una misma madre durante el período estudiado. Todos los binomios fueron analizados en forma separada aún cuando correspondieran a una misma madre. Las principales características clínicas de las madres se resumen en la Tabla 1. Es destacable que en la mitad de los casos el diagnóstico de infección por VIH se hizo previo al embarazo, con una mediana de 24 (rango: 1-168) meses de antelación entre el diagnóstico de la infección por VIH y el embarazo. En 40 embarazadas (42,5\%), el motivo de la toma del ELISA VIH fue el embarazo, ya fuese la gestación en curso o una previa (Figura 2). Respecto a co-infecciones relevantes, dos madres tuvieron durante su embarazo una infección aguda por Toxoplasma gondii sin manifestaciones clínicas (en una se demostró conversión de $\operatorname{IgM}$ para $T$. gondii de dudoso a positivo en el lapso de dos meses y la otra tuvo IgM e IgG positivas en un solo control sin seguimiento posterior) y otra paciente era portadora de virus de hepatitis B con un antígeno de superficie positivo, sin evidencias clínicas ni de laboratorio de enfermedad hepática. Ninguna paciente desarrolló infecciones oportunistas asociadas al VIH durante su gestación.

El $19,1 \%$ de las madres (18/94) estaba recibiendo TARV previa al embarazo: 4 con nevirapina, 5 con efavirenz y 9 con IP. De las restantes, 20 (21,3\%) iniciaron TARV durante el embarazo por requerimiento materno y $53(56,4 \%)$ iniciaron TARV como parte del PPTV. La mediana de inicio de TARV fue a las 23 semanas de embarazo (rango: 0-38). Catorce pacientes (14,9\%) recibieron monoterapia, $8(8,5 \%)$ biterapia y $69(73,4 \%)$ triterapia, 61 de las cuales incluyó IP (Tabla 2).

Al momento del parto, el 75,5\% de las mujeres embarazadas (71 pacientes) contaba con estudio reciente, realizado dentro del mes previo al parto, de recuento de linfocitos T CD4 con una mediana de 341 céls $/ \mathrm{mm}^{3}$ (rango 76-952); por otra parte, 71,3\% (67 pacientes) de las mujeres gestantes tenían estudio de carga viral concomitante, de las cuales $28,7 \%$ (27 pacientes) tenía menos de 80 copias ARN/ml y 40,4\% (38 pacientes) tenía menos de 1.000 copias ARN/ml.

De los 94 partos realizados, $83(88,3 \%)$ se resolvieron por cesárea electiva, $11(11,7 \%)$ por cesárea de urgencia y $1(1,1 \%)$ por vía vaginal. Hubo 14 recién nacidos $(14,6 \%)$ de pre-término sin observarse correlación con el uso materno de IP: $16,4 \%$ (10) recién nacidos expuestos al uso materno de IP versus $12,1 \%$ (4) recién nacidos 
no expuestos al uso materno de IP $(\mathrm{p}=0,71)$. Por otra parte, 20 recién nacidos $(20,8 \%)$ presentaron retardo de crecimiento intrauterino; sin embargo, tampoco se encontró relación con el uso materno de IP: 23\% (14) recién nacidos expuestos al uso materno de IP versus $17,1 \%$ (6) no expuestos al uso materno de IP $(p=0,67)$. El $94,8 \%$ de los niños no recibió lactancia materna y $66,7 \%$ recibió protocolo de prevención de TV completo, según las guías MINSAL ${ }^{15}$ (75\% sólo con zidovudina, 12,5\% con zidovudina + lamivudina y $12,5 \%$ con zidovudina + nevirapina). En dos recién nacidos hubo TV, con una tasa de 2,08\%. De los dos niños infectados, uno recibió PPTV completo y el otro incompleto por consulta tardía (4 días previos al parto) de su madre seropositiva conocida.

Las morbilidades presentes en los recién nacidos se describen en la Tabla 3. Ochenta y dos niños $(85,4 \%)$ presentaron anormalidades clínicas o de laboratorio posiblemente atribuibles a la exposición a la TARV; en 47 de ellos $(57,3 \%)$ co-existieron dos o más anormalidades. La anemia fue el evento adverso más frecuente, detectándose en 68 recién nacidos $(70,8 \%)$, con una mediana de hematocrito de 26\% (rango: 17-30). Lo más frecuente fue la presentación al mes de vida, en $58,8 \%$ de los casos (40/68); sin embargo, en 20,6\% (14/68) fue más precoz (entre el período de recién nacido inmediato y los 21 días de vida) y en igual porcentaje en mayores de un mes y hasta dos meses. En cuanto a la graduación, lo más frecuente fue anemia grado 2 en 36 niños $(53 \%)$ y anemia grado 3 en 29 niños (42,6\%). Seis niños requirieron transfusión de eritrocitos, 4 de ellos en las primeras horas de vida y 2 al mes de edad; en 1 caso se usó eritropoyetina intravenosa.

El segundo evento adverso más frecuente fueron las alteraciones metabólicas presentes en 51 niños (53,1\%), 11 de los cuales presentó más de una alteración metabólica. La más frecuente fue la hiperlactacidemia: 40 niños $(41,7 \%)$ tuvieron valores de lactato en sangre superiores al normal máximo $(2,1 \mathrm{mmol} / \mathrm{l}), 28(29,2 \%)$ sin acidosis y $12(12,5 \%)$ con acidosis. Más de $90 \%$ se pesquisó entre la primera y tercera semana de vida (en un recién nacido se detectó el primer día de vida), alcanzando valores $\geq 5$ mmol/1 en 17 niños (42,5\%): en 14 niños hubo toxicidad grado 3 y en 3 niños fue grado 4 . La anormalidad duró en promedio $31,7 \pm 25,4$ días y fueron tratados con bicarbonato en promedio $25,9 \pm 17,3$ días. Sólo dos niños tuvieron síntomas relacionados: bajo incremento ponderal en un caso e hipertonía en otro. En nueve casos se asoció a hiperkalemia y en 28 se presentó junto a anemia. En otros nueve niños se encontró acidosis metabólica $(9,4 \%)$ que duró en promedio $29 \pm 14,1$ días. Todos fueron tratados con bicarbonato en promedio 32,4 $\pm 11,1$ días hasta la normalización de los parámetros. En 14 niños $(14,6 \%)$ se encontró hiperkalemia, con un valor promedio de 5,4 $\mathrm{meq} / 1 \pm 0,58$. Cuatro de ellos evolucionaron con un cuadro compatible con acidosis tubular renal tipo IV hiperkalé-

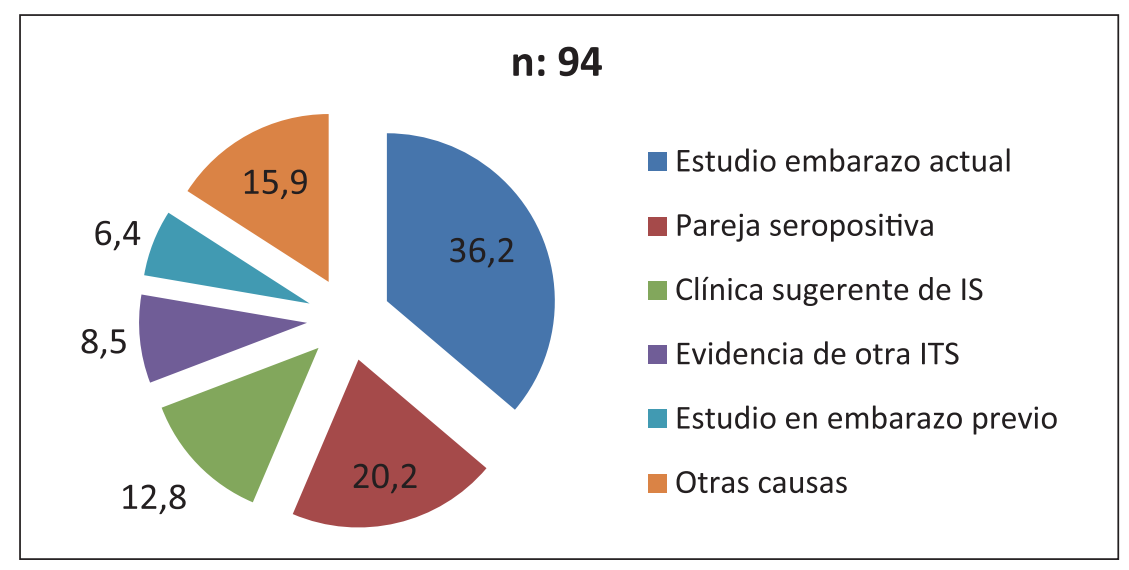

Figura 2. Motivo de toma del examen de ELISA VIH de las 94 embarazadas analizadas en esta serie.

Tabla 2. Esquemas e indicación de terapia anti-retroviral en las mujeres embarazadas con infección por VIH

$\begin{array}{lrr}\text { Esquemas e indicación de TARV } & \mathbf{n} & \% \\ \text { TARV iniciada pre-embarazo } & 18 & \mathbf{1 9 , 1} \\ \text { Triterapia con nevirapina } & 4 & 4,2 \\ \text { Triterapia con efavirenz } & 5 & 5,3 \\ \text { Triterapia con inhibidores de proteasa } & 9 & 9,6 \\ \text { TARV iniciada durante el embarazo. Justificación para el inicio } & 73 & \mathbf{7 7 , 7} \\ \text { Requerimiento materno } & 20 & 21,3 \\ \text { Requerimiento fetal } & 53 & 56,4 \\ \text { Esquemas de TARV utilizados en el embarazo } & 91 & 96,8 \\ \text { Monoterapia } & 14 & 14,9 \\ \text { Biterapia } & 8 & 8,5 \\ \text { Triterapia sin inhibidores de proteasa }{ }^{\circledR} & 8 & 8,5 \\ \text { Triterapia con inhibidores de proteasa }{ }^{\mathbf{Q}^{*}} & 61 & 64,9\end{array}$

NOTA: ${ }^{8}$ Columna vertebral: zidovudina/lamiduvina 64 , abacavir/lamiduvina 2 , didanosina/lamiduvina 2, didanosina/staduvina 1; *opinavir/ritonavir 31, nelfinavir 18 , atazanavir/ritonavir 2, saquinavir/ ritonavir 4, indinavir 6 .

Tabla 3. Anormalidades encontradas en los recién nacidos expuestos a TARV

\begin{tabular}{lcc} 
Morbilidad en recién nacidos & $\mathbf{n}$ & $\%$ \\
Alteraciones hematológicas & 68 & 70,8 \\
Anemia & 1 & 1 \\
Neutropenia & 1 & 1 \\
Trombocitopenia & & \\
Alteraciones metabólicas & 28 & 29,2 \\
Hiperlactacidemia sin acidosis & 14 & 14,6 \\
Hiperkalemia & 12 & 12,5 \\
Hiperlactacidemia con acidosis & 9 & 9,4 \\
Acidosis metabólica & 1 & 1 \\
Hipercalcemia & 20 & 20,8 \\
Retardo del crecimiento intrauterino & 14 & 14,6 \\
Prematurez & 4 & 4,2 \\
Malformaciones cardíacas congénitas & 2 & 2,1 \\
Retraso del crecimiento pondo-estatural & 2 & 2,1 \\
\hline Hipertonía & &
\end{tabular}


Tabla 4. Factores de riesgo para el desarrollo de alteraciones metabólicas en lactantes nacidos de madres con infección por VIH

\begin{tabular}{|c|c|c|c|c|c|}
\hline Variables & $\begin{array}{c}\text { Desarrollo de } \\
\text { alteraciones } \\
\text { metabólicas en RN } \\
\%(n=51)\end{array}$ & $\begin{array}{l}\text { Sin desarrollo de } \\
\text { alteraciones } \\
\text { metabólicas en RN } \\
\%(n=49)\end{array}$ & $\stackrel{p}{p}$ Análisis univariado & $\begin{array}{c}\text { OR }(95 \% \mathrm{Cl}) \\
\text { Análisis } \\
\text { multivariado }\end{array}$ & p \\
\hline \multicolumn{6}{|l|}{ Factores de la madre } \\
\hline Edad, promedio (SD) & $28,2 \pm 6,7$ & $26,6 \pm 5,7$ & 0,21 & - & - \\
\hline Co-morbilidades & $15,1 \%$ & $19 \%$ & 0,78 & - & - \\
\hline Drogadicción & $7,5 \%$ & $14,3 \%$ & 0,32 & - & - \\
\hline CD4 basales $<200$ céls $/ \mathrm{mm}^{3}$ & $46,9 \%$ & $35,7 \%$ & 0,47 & - & - \\
\hline Etapa SIDA al diagnóstico & $48 \%$ & $41,4 \%$ & 0,64 & - & - \\
\hline Inicio tardío de control de embarazo* & $52,9 \%$ & $44,7 \%$ & 0,52 & - & - \\
\hline Inicio de TARV previo a semana 24 de gestación & $43,4 \%$ & $57,1 \%$ & 0,21 & - & - \\
\hline Uso de inhibidores de proteasa durante el embarazo & $84,9 \%$ & $41,9 \%$ & $<0,001$ & $4 \quad(1,58-10,12)$ & 0,003 \\
\hline Uso de ritonavir durante el embarazo & $58,5 \%$ & $16,3 \%$ & $<0,001$ & $1,03(0,5-2,02)$ & 0,94 \\
\hline \multicolumn{6}{|l|}{ Factores del RN } \\
\hline Prematurez & $25 \%$ & $4,7 \%$ & 0,009 & $1,3(0,65-2,64)$ & 0,45 \\
\hline Retardo de crecimiento intrauterino & $31,9 \%$ & $9,3 \%$ & 0,01 & $1,36(0,7-2,71)$ & 0,36 \\
\hline Sexo & $50 \%$ & $55,8 \%$ & 0,68 & - & - \\
\hline Monoterapia con zidovudina & $88,7 \%$ & $58,1 \%$ & 0,001 & $0,8 \quad(0,29-2,2)$ & 0,66 \\
\hline Biterapia antiretroviral ${ }^{\&}$ & $11,3 \%$ & $41,9 \%$ & 0,001 & $0,15(0,02-1,36)$ & 0,09 \\
\hline Uso de lamivudina en RN & $1,9 \%$ & $25,6 \%$ & 0,001 & $0,15(0,02-1,13)$ & 0,06 \\
\hline
\end{tabular}

mica y fueron tratados con bicarbonato y poliestireno de sulfato de sodio (Kayexalate $\left.{ }^{\circledR}\right)$; en un caso ésta se resolvió a los 17 meses y 3 permanecen en tratamiento a los 8,10 y 22 meses de edad, en el resto de los niños la anormalidad duró en promedio $28,8 \pm 2,5$ días.

Otras de las morbilidades detectadas fueron: hipertonía sin otras manifestaciones neurológicas ( 2 casos) asociada a acidosis metabólica en 1 niño, retraso de crecimiento pondo-estatural ( 2 casos) asociado a hiperkalemia en un niño y en otro a acidosis metabólica, hipercalcemia, neutropenia grado 1 al mes y trombocitopenia grado 2 al nacer en un caso cada una. Estas últimas tres anormalidades se asociaron a anemia.

El estudio de infecciones transplacentarias demostró infección por citomegalovirus en 8 de 48 recién nacidos estudiados (16,7\%); sin embargo, los exámenes efectuados (hemograma, pruebas hepáticas, ecografía cerebral y/o TAC cerebral, fondo de ojo y emisiones otoacústicas) descartaron enfermedad citomegálica. En ninguno de los hijos de madres que tuvieron infección aguda por T. gondii durante el embarazo ni de aquella portadora de hepatitis B se confirmó infección congénita.

En 4 niños (4,2\%) se encontró malformación cardíaca congénita: comunicación interventricular (4), en uno asociada a comunicación interauricular y en otro con ductos arterioso persistente. Otro niño tuvo foramen oval permeable no considerado malformación.

Variables candidatas examinadas como factores de riesgo para el desarrollo de alteraciones metabólicas en los recién nacidos son mostradas en la Tabla 4. El análisis multivariado mostró que la exposición materna al uso de IP fue un factor independiente para el desarrollo de alteraciones metabólicas en los recién nacidos (OR 4 [1,58-10,12], p 0,003).

\section{Discusión}

En Chile, como en el resto del mundo, la pandemia de infección por VIH/SIDA continúa en aumento. Anualmente, cerca de 2.000 personas lo contraen, constituyendo una prevalencia entre 0,2 y $0,3 \%$ en la población general ${ }^{15}$. Desde 1987, año de diagnóstico del primer caso pediátrico y hasta diciembre de 2011 se han diagnosticado 326 casos de niños hijos de madres infectadas con VIH en el país ${ }^{2}$.

En nuestro país, las notificaciones de TV alcanzan al $0,6 \%$ del total de casos reportados de VIH/SIDA en el quinquenio 2007-2011 ${ }^{17}$, y considerando que más de $90 \%$ de los casos de infección por VIH en pediatría ocurre a través de este mecanismo de transmisión ${ }^{18}$, los esfuerzos deben concentrarse en la pesquisa de las mujeres embarazadas sero positivas para efectuar la profilaxis anti-retroviral que reduzca la trasmisión vertical a valores cercanos a cero.

Desde 1997, se incorporan en Chile las "Recomendaciones para la prevención de la TV del VIH/SIDA"19 con tamizaje materno y parto por cesárea, y en 2005 el 
protocolo de prevención de la TV. Como consecuencia y posterior a la incorporación de la infección por VIH/ SIDA como patología GES este mismo año, se detectó en el CADSR un aumento significativo de las mujeres embarazadas que accedieron a la profilaxis ya que el equipo de salud capacitado comenzó a ofrecer el test de ELISA para VIH lo que derivó en un aumento sostenido de la realización de este examen entre los años 2006 y 2008. Asimismo, la oportunidad del diagnóstico permitió aplicar el protocolo de prevención de TV en forma completa confirmándose la efectividad de la TARV en prevenir la TV.

Nos parece que nuestra serie es representativa de la población nacional de mujeres embarazadas al abarcar a un amplio sector de la zona suroriente de Santiago, con una población asignada de 1,6 millones de personas, de las cuales alrededor de $74 \%$ está inscrita para la atención en el sistema de salud primaria. Dentro de las características maternas, encontramos que al igual que lo descrito en otras series nacionales, la vía de transmisión es predominantemente sexual ${ }^{20}$ y aunque, por ser una población joven, presenta un bajo porcentaje de co-morbilidades, destaca un número no despreciable de madres con patología psiquiátrica, lo que ha sido descrito en otras series de la literatura médica ${ }^{21}$ y que tiene importancia pues puede dificultar la adherencia a la TARV. En este grupo de mujeres embarazadas, no se encontraron grandes diferencias respecto a lo descrito en otras series nacionales. El objetivo de lograr una carga viral indetectable o menor a 1.000 copias ARN/ $\mathrm{ml}$, hecho relevante a la hora de evitar la TV, se pudo verificar en $69,1 \%$ de las pacientes, lo que es discretamente mayor a lo descrito en otras series nacionales donde se alcanza hasta en $64 \%$ de las pacientes ${ }^{22}$.

La TARV durante el embarazo y período perinatal ha sido exitosa en reducir de forma importante la TV del VIH; sin embargo, puede producir efectos colaterales en el recién nacido. Hay estudios contradictorios respecto al riesgo de prematurez y bajo peso al nacer secundario al uso de TARV, específicamente por el uso de IP durante el embarazo $^{23-25}$. En nuestra serie no se evidenció aumento de riesgo de prematurez o bajo peso al nacer en relación a la exposición a IP. Por otra parte, varios estudios demuestran que la TARV no aumenta el riesgo de malformaciones congénitas en el recién nacido ${ }^{25}$. La tasa de malformaciones congénitas encontrada en esta cohorte $(4,2 \%)$ no difiere estadísticamente de la reportada en otros estudios que evalúan malformaciones en población general como los estudios del CDC (2,66\%), Chile (2,81\%) o ECLAMC (Estudio Colaborativo Latino Americano de Malformaciones Congénitas $(2,63 \%)^{26-28}$.

En cuanto a las infecciones transplacentarias, en este grupo se encontró una incidencia de infección por CMV superior a la descrita en la literatura médica $(16,7 \%$ versus $4,5 \%)^{29,30}$ probablemente por el bajo nivel socioeconómico de la población de mujeres embarazadas. Este resultado sugiere la necesidad de implementar el estudio de CMV con shell vial en orina dentro de los primeros 15 días de vida.

La toxicidad de los anti-retrovirales puede afectar tanto al feto como al recién nacido. El primer estudio de prevención de TV (PACTG 076) usando zidovudina, documentó toxicidad a corto plazo traducida en anemia transitoria $^{31}$. En esta serie de niños expuestos, éste fue el efecto colateral más frecuente $(70,8 \%)$ siendo en general de intensidad moderada; sin embargo, 6 niños requirieron transfusión de eritrocitos, en su mayoría, en las primeras semanas de vida y en 21,6\% la anemia fue diagnosticada antes del mes de vida lo que obliga a hacer controles hematológicos desde el nacimiento. En todos los casos se recuperó una vez suspendida la TARV, lo que concuerda con lo descrito en la literatura médica ${ }^{6-8,31}$, sin embargo, en algunos casos se prolongó hasta los 2 meses de edad, situación similar a la ocurrida en los recién nacidos tratados con el protocolo PACTG $076^{31}$.

Los inhibidores de la transcriptasa reversa análogos de nucleósidos (INTR) son desoxinucleósidos, que son fosforilados dentro de la célula y transformados en dinucleótidos (dNTP) por lo que podrían actuar como inhibidores o substratos de las ADN polimerasas (ADNp) humanas. La ADNp gama es la única implicada en la replicación del ADN mitocondrial (ADNmt) por lo que la acción inhibitoria de los INTR sobre esta enzima puede afectar a la replicación y función mitocondrial, interfiriendo con la síntesis de las proteínas de la cadena respiratoria y desviando la producción de energía a la vía alternativa con aumento en la producción de ácido láctico y acúmulo de ácidos grasos. La depleción del ADNmt ocurre en diversos órganos, como corazón, hígado, músculo esquelético, nervios periféricos, placenta e incluso sangre de cordón. No todos los INTR tienen la misma potencia para inhibir la síntesis de ADNmt (zalcitabina > didanosina y estavudina $>$ lamivudina $>$ zidovudina $>$ abacavir). La elevación de lactato en sangre puede ser moderada $(>2,1-5)$ o intensa ( $\geq 5 \mathrm{~mm} / \mathrm{l})$, sintomática o asintomática y puede llegar a ser fatal. La veracidad del resultado del examen está sujeta a ciertas condiciones: extracción de la muestra sin ligadura para evitar la venoectasia, depósito en tubo heparinizado previamente refrigerado y transporte en hielo para ser procesado antes de 20 min de su extracción. La elevación de lactato y la acidosis láctica están descritas en varias series de recién nacidos expuestos a INTR.

La prevalencia de hiperlactacidemia varía en las distintas series y en la mayoría de los reportes la toxicidad no es clínicamente relevante. Por otra parte, los factores asociados descritos son variados tanto de la madre (edad, consumo de drogas, estadío clínico de la enfermedad, carga viral, TARV, entre otros) como del recién nacido (prematurez, peso de nacimiento, tipo de anti-retroviral, 
duración de exposición, entre otros). Un estudio reportó valores anormales de lactato tomados en al menos una muestra, en 35 de 38 niños (92\%), sin relación con el tipo de anti-retroviral, duración del tratamiento, gravedad de la enfermedad materna y uso de drogas. El 26\% tuvo hiperlactacidemia intensa y 2 niños fueron sintomáticos. La mayoría revirtió al suspender el INTR; sin embargo, $6,1 \%$ lo mantuvo elevado hasta los 7 meses de edad ${ }^{32}$. Otro reporte, con una serie de niños españoles expuestos a TARV (PPTV) similar en tipo de anti-retroviral y tiempo de exposición a nuestros pacientes, confirmó estos hallazgos encontrando hiperlactacidemia en 63 de 127 niños expuestos $(49,6 \%)$ y niveles superiores de lactato al compararlos con controles en tomas sucesivas hasta los 12 meses de edad, con retraso del desarrollo psicomotor en 3 niños, asociado a niveles superiores a $4 \mathrm{~mm} / \mathrm{s}^{32}$. En este estudio no se encontró diferencia significativa entre la mayoría de los anti-retrovirales utilizados, a excepción de la didanosina, la que presentó un mayor porcentaje de hiperlactacidemia en los niños expuestos a ésta ${ }^{33}$. Otro estudio efectuado en 623 niños españoles confirmó una importante prevalencia de hiperlactacidemia (48-51\%, a los 15 días y 45 días, respectivamente) y no encontró diferencias significativas entre la mayoría de los anti-retrovirales estudiados, excepto una mayor incidencia con el uso de abacavir ${ }^{34}$. Algunos estudios demuestran que la prevalencia de hiperlactacidemia depende del tiempo de exposición, siendo significativamente mayor, cuando es más largo ${ }^{34}$. Algunos autores no recomiendan la medición rutinaria de lactato en sangre en recién nacidos expuestos a TARV en PPTV de corta duración. Un estudio efectuado en África no demostró diferencia significativa en la prevalencia de hiperlactacidemia en el grupo expuesto ( 8 semanas durante el embarazo y hasta 7 días de vida) comparado con el control $(13,1$ vs $14,3 \%, p=0,84)$, ningún paciente fue sintomático y $90 \%$ tuvo mediciones normales a los 3 meses de edad $^{35}$. En nuestra serie, $41,7 \%$ de los niños tuvo hiperlactacidemia, en casi la mitad fue intensa y en 12 asociada a acidosis. El análisis multivariado mostró que la exposición materna al uso de IP fue un factor independiente para el desarrollo de alteraciones metabólicas en los recién nacidos. Aun cuando dentro de las reacciones adversas más frecuentemente descritas para los IP no se encuentran las alteraciones metabólicas y más específicamente, la hiperlactacidemia, sí hay series grandes de pacientes que han encontrado asociación significativa entre el uso de ritonavir (como parte del refuerzo del IP) e hiperlactacidemia, aunque la explicación fisiopatológica de esta asociación no se ha logrado $\operatorname{aclarar}^{36,37}$. Dada la naturaleza retrospectiva de nuestra serie, es posible que nuestros resultados tengan sesgos por la falta de evaluación de otras variables, sobre todo maternas, para las cuales se ha descrito asociación con el desarrollo de hiperlactacidemia como son el incremento del índice cintura/cadera materno o la alteración de enzimas hepáticas maternas. No se analizó la variable exposición materna a didanosina debido a que ésta se utilizó sólo en 3 casos.

Catorce niños presentaron hiperkalemia (14,6\%), 4 de ellos evolucionaron con un cuadro compatible con acidosis tubular renal tipo IV hiperkalémica que se mantuvo en uno hasta los 17 meses de edad; los otros aún están en tratamiento con bicarbonato y resinas de intercambio iónico (Kayexalate ${ }^{\circledR}$ ) a $\operatorname{los} 8,10$ y 22 meses de edad. Aunque no hay reportes al respecto, esta podría ser la expresión a largo plazo de toxicidad mitocondrial, lo que nos hace recomendar la evaluación de laboratorio de los niños expuestos a anti-retrovirales durante el período de profilaxis y a más largo plazo en aquellos en los que se demuestren alteraciones de los exámenes.

Un estudio francés reportó disfunción mitocondrial en $0,45 \%$ (8 de 1.754 niños expuestos a zidovudina y lamivudina) y demostró evidencia de disfunción mitocondrial persistente en biopsia muscular en 7 de 2.644 niños expuestos versus ninguno de 1.748 no expuestos. Dos niños presentaron síntomas neurológicos graves y fallecieron 9 . Posteriormente el mismo grupo reportó una incidencia de $0,2 \%{ }^{38}$. En nuestros pacientes no se detectaron síntomas en los controles habituales, salvo en 2 que evolucionaron con retraso pondo estatural y en otros 2 que tuvieron hipertonía la que regresó durante el período de control; sin embargo no se efectuó control neurológico rutinario en todo el grupo estudiado. Si bien la incidencia de disfunción mitocondrial es baja, existe la posibilidad de compromiso y deterioro del sistema nervioso central y periférico lo que hace necesario que se efectúen evaluaciones neurológicas acuciosas y periódicas. El diagnóstico de certeza de toxicidad mitocondrial se hace mediante la cuantificación del ADNmt o al menos un examen histológico en búsqueda de alteraciones ultra estructurales de la mitocondria y acúmulo de vacuolas grasas; actualmente sólo es posible efectuar niveles sanguíneos frente a la sospecha clínica. Durante los últimos años, se han realizado nuevos estudios que intentan identificar marcadores de toxicidad mitocondrial desde sangre materna, de cordón y de sangre periférica de recién nacido, entre los cuales se han medido niveles de ARN y ADN mitocondrial, apoptosis, síntesis de proteína mitocondrial materna y fetal, entre otros, pero con resultados disímiles ${ }^{39,40}$.

De acuerdo a nuestros hallazgos y considerando el momento de presentación de las anormalidades de laboratorio, nos parece recomendable efectuar un seguimiento de los recién nacidos hijos de madre con infección por VIH expuestos a TARV que contemple: evaluación clínica y exámenes de laboratorio (hemograma, recuento de plaquetas, lactato plasmático, electrolitos plasmáticos, 
gases venosos, pruebas hepáticas) al nacer y al mes de vida con el fin de pesquisar anormalidades y tratarlas oportunamente, al mismo tiempo es imprescindible que el recién nacido sea controlado por el médico tratante en forma periódica y sea evaluado por un neurólogo para detectar síntomas posiblemente relacionados a toxicidad por anti-retrovirales.

\section{Conclusión}

El aumento sostenido de embarazos en mujeres con infección por VIH obliga a los equipos de salud a verificar la aplicación del protocolo de prevención de TV en forma completa considerando su probada efectividad. En nuestra serie, la TARV demostró ser efectiva en reducir la TV logrando tasas similares a $2 \%$. Sin embargo, ésta no está exenta de efectos adversos en los recién nacidos expuestos. En el grupo estudiado se encontraron los más frecuentemente descritos en la literatura: anemia y complicaciones metabólicas. Debido a su alta frecuencia es recomendable la implementación de programas de seguimiento de estos pacientes para prevenir secuelas posteriores.

Agradecimientos: A Daniel Ernst por su colaboración en la recolección de datos.

\section{Resumen}

La terapia anti-retroviral (TARV) es efectiva en disminuir la transmisión vertical (TV) del VIH, pero no está exenta de efectos adversos en los recién nacidos: riesgo de prematurez, bajo peso al nacer, alteraciones metabólicas y otros. Pese a lo relevante del tema, existen pocos datos nacionales que analicen el problema. Realizamos un estudio observacional, retrospectivo, de una serie de binomios madre infectada por VIH/hijo atendidos entre los años 1995 y 2010. Se analizaron 94 embarazos y 96 hijos (2 embarazos gemelares). La tasa de TV fue 2,1\%. De los recién nacidos, $85,4 \%$ presentó efectos adversos atribuibles a la exposición a TARV destacando la presencia de anemia (70,8\%) y alteraciones metabólicas varias [hiperlactacidemia sin acidosis $(29,2 \%)$, acidosis láctica $(12,5 \%)$, hiperkalemia $(14,6 \%)$ y acidosis metabólica $(9,4 \%)$. La exposición materna al uso de IP demostró ser un factor de riesgo independiente para el desarrollo de alteraciones metabólicas en los recién nacidos (OR 4 [1,58-10,12], p 0,003). En nuestra serie, la TARV demostró ser efectiva en reducir la TV. Sin embargo, los recién nacidos expuestos presentaron alta frecuencia de efectos adversos, por lo que es recomendable la implementación de programas de seguimiento de estos pacientes para prevenir secuelas.

\section{Referencias bibliográficas}

1.- Quinn T, Overbaugh J. HIV/AIDS in women: an expanding epidemic. Science 2005; 308: 1582-3.

2.- Díaz J. Norma conjunta de prevención de la transmisión vertical del VIH y la sífilis. Rev Chil Infectol 2013; 30: 259-302.

3.- Pan American Health Organization. 2012 Progress Report. Elimination of mother-to-child transmission of VIH and congenital syphilis in the Americas. Washington D.C., Panamerican Health Organization, 2013.

4.- Chávez A, Álvarez A, Wu E. Transmisión vertical de la infección por virus de la inmunodeficiencia humana: Impacto de la aplicación del protocolo ACTG 076 en Chile. Rev Chilena Infectol 2000; 17: 297-301.

5.- Chávez A, Álvarez A, Wu E, Peña A, Vizueta E, Comité Nacional del SIDA Pediátrico. Evolución de la transmisión vertical de la infección por virus de la inmunodeficiencia humana en Chile. Rev Chilena Infectol 2007; 24: 368-71.

6.- Le Chenadec J, Mayaux MJ, GuihenneucJouyaux C, Blanche S, Enquete Perinatale Francaise Study Group. Perinatal antiretroviral treatment and hematopoiesis in HIV-uninfected infants. AIDS 2003; 17: 2053-61.
7.- Feiterna-Sperling $\mathrm{C}$, Weizsaecker $\mathrm{K}$, Bührer C, Casteleyn S, Loui A, Schmitz T, et al. Hematologic effects of maternal antiretroviral therapy and transmission prophylaxis in HIV-1-exposed uninfected newborn infants. J Acquir Immune Defic Syndr 2007; 45: 43-51.

8.- Pacheco S E, McIntosh K, Lu M, Mofenson L M, Díaz C, Foca M, et al. Effect of perinatal antiretroviral drug exposure on hematologic values in HIV-uninfected children: An analysis of the women and infants transmission study. J Infect Dis 2006; 194: 1089-97.

9.- Blanche S S, Tardieu M, Rustin P, Slama A, Barret B, Firtion G, et al. Persistent mitocondrial dysfunction and perinatal exposure to antiretroviral nucleoside analogues. Lancet 1999; 354: 1084-9.

10.- French Perinatal Cohort Study. Risk of early febrile seizure with perinatal exposure to nucleoside analogues. Lancet 2002; 359: 583-4.

11.- Suy A, Martínez E, Coll O, Lonca M, Palacio M, De Lazzari E, et al. Increased risk of pre-eclampsia and fetal death in HIV-infected pregnant women receiving highly active antiretroviral therapy. AIDS 2006; 20: 59-66

12.- Thorne C, Newell M L. Safety of agents used to prevent mother-to-child transmission of HIV: is there any cause for concern? Drug Saf 2007; 30 203-13. [review].

13.- Ministerio de Salud de Chile. Manual de procedimientos para la detección y diagnóstico de la infección por VIH, MINSAL Chile, 2010.

14.- Ministerio de Salud de Chile. CONASIDA. Estrategia de atención integral a personas que viven con VIH/SIDA. Programa Nacional de Prevención y Control del VIH/SIDA, 2006; 1-13.

15.- Ministerio de Salud de Chile. Guía Clínica Síndrome de Inmunodeficiencia Adquirida VIH/ Sida. Santiago: MINSAL $2010 \mathrm{http}: / / \mathrm{www}$. minsal.cl/portal/url/item/7220fdc4340c44a9e04 001011f0113b9.pdf (accedido el 31 de enero de 2013).

16.- Guidelines for Blood Drawn for Research Purposes in the Clinical Center NIH Clinical Center. internal.cc.nih.gov/policies/PDF/M95-9. pdf (accedido el 14 de noviembre de 2011).

17.- Informe Nacional "Evolución VIH SIDA, Chile 1984-2011, MINSAL, agosto de 2012.

18.- Chávez A, Álvarez AM, Wu E y Comité Nacional de SIDA Pediátrico, Sociedad Chilena de Pediatría. Transmisión vertical de la infección por virus de inmunodeficiencia humana: impacto de la aplicación del protocolo ACTG076 en Chile. Rev Chilena Infectol 2000; 17: 297-301. 
19.- Ministerio Salud, Programa Salud de la mujer. Chile, 1997.

20.- Valdés E, Sepúlveda A, Candia P, Sepúlveda C, Lattes K. VIH/SIDA: Comportamiento epidemiológico de la transmisión vertical en el contexto general de la infección en Chile. Rev Chil Obstet Ginecol 2011; 76: 52-7.

21.- James M E, Rubin C P, Willis S E. Drug abuse and psychiatric findings in HIV-seropositive pregnant patients. Gen Hosp Psychiatry 1991; 13: 4-8.

22.- Paredes A, Córdova V, Cofré J, Lattus J, Ballestero J. Manejo y resultados en la resolución de pacientes embarazadas $\mathrm{VIH} /$ SIDA positivas en el servicio de Obstetricia y Ginecología Hospital Santiago Oriente Dr. Luis Tisné Brousse. Rev Chil Obstet Ginecol 2012; 7: 11-8.

23.- Rosso R, Di Biagio A, Gotta C, Transino S, Bentivoglio G and Viscoli C. Use of tenofovir in a cohort of HIV-infected pregnant women and their infants. Abstract, AIDS 2010, Viena. THPEO 148.

24.- Siberry G K, Williams P L, Méndez H, Seage G, Jacobson D L, Hazra R, et al, for the Pediatric HIV/AIDS Cohort. Safety of tenofovir use during pregnancy: associations with low birth weight and early growth in HIV-exposed uninfected infants. $2^{\text {nd }}$ International Workshop on HIV Pediatrics. 16-17 july 2010, Vienna, Austria. Abstract, AIDS 2010, Viena WEAXO 103.

25.- Bekerman K, Albano J, Martínez-Tristani M, Seekins D, Storfer S, David N, et al. Preterm birth (PTB), low birth wiegth (LBW) and fetal antiretroviral (ARV) exposure: Gestacional age (EGA) and birth weight data from 10022 singleton live births (LB) reported to the Antiretroviral Pregnancy Registry (APR) 1989 through 31 January 2009. $2^{\text {nd }}$ International Workshop on HIV Pediatrics. 16-17 july 2010, Vienna, Austria. Abstract, AIDS 2010, Viena. WEAXO105.

26.- Antiretroviral Pregnancy Registry Steering
Committee. (2010) Antiretroviral pregnancy registry international interim report for 1 January 1989 through 31 January 2010. http:// www.APRegistry.com (accedido el 26 de noviembre de 2010).

27.- Correa-Villasen A, Cragan J, Kucik J, O'Leary L, Siffel C, Williams L. The Metropolitan Atlanta Congenital Defects Program: 35 Years of Birth Defects Surveillance at the Centers for Disease Control and Prevention. Birth Defects Research (Part A) 2003; 67: 617-24.

28.- Nazer J, Aravena T, Cifuentes L. Malformaciones congénitas en Chile. Un problema emergente (período 1995-1999). Rev Med Chile 2001; 129: 895-904.

29.- Marín G, Fernández M, González M, Saavedra J, Barajas V, Rojo P, et al. Infección congénita por citomegalovirus en hijos de madres infectadas por el VIH. Anales de Pediatría 2005; 62: 38-42.

30.- Mostad S B, Kreiss J K, Ryncarz A, Overbaugh J, Mandaliya K, Chohan B, et al. Cervical shedding of herpes simplex virus and cytomegalovirus throughout the menstrual cycle in women infected with human immunodeficiency virus type 1 . Am J Obstet Gynecol 2000; 183: 948-55.

31.- Connor E M. Reduction of maternal-infant transmission of human immunodeficiency virus type 1 with zidovudine treatment. Pediatric AIDS Clinical Trials Group Protocol 076 Study Group. N Engl J Med 1994; 331 : 1173-80

32.- Alimenti A, Burdge D, Ogilvie G, Money D, Forbes J. Lactic academia in human inmunodeficient virus-uninfected infants exposed to perinatal antiretroviral therapy. Pediatr Infect Dis J 2003; 22: 782-9.

33.- Noguera, A, Fortuna C, Muñoz-Almagro C, Sánchez E, Vilaseca MA, Artuch R, Pou J, et al. Hyperlactatemia in human immunodeficiency virus-uninfected infants who are exposed to antiretrovirals. Pediatrics 2004; 114; e598-603.

34.- Fernández M, Bellón J, Ramos J, GonzálezTomé M, Guillén S, Navarro N, et al. Exposición a antirretrovirales y toxicidad mitocondrial en una cohorte de niños no infectados hijos de madres VIH positivas. An Pediatr (Barc) 2010; 73: 180-8.

35.- Koumavi Ekouevi D, Touré R, Becquet R, Viho I, Sakarovitch C, Rouet F, et al. Serum lactate levels in infants exposed peripartum to antiretroviral agents to prevent mother-to-child transmission of HIV: Agence Nationale de Recherches Sur le SIDA et les Hépatites Virales 1209 Study, Abidjan, Ivory Coast. Pediatrics 2006; 118: e1071-7.

36.- Imhof A, Lefergerber B, Günthard H, Haupts S, Weber R and the Swiss HIV Cohort Study. Risk factors for and outcome of hyperlactatemia in HIV-infected persons: Is there a need for routine lactate monitoring? Clin Infect Dis 2005; 41: 721-8.

37.- Huynh T K, Lüttichau H R, Roge B T, Gerstoft J. Natural history of hyperlactataemia in human immunodeficiency virus-1 infected patients during highly active antiretroviral therapy. Scand J Infect Dis 2003; 35: 62-6.

38.- Barret B, Tardieub M, Rustinc P, Lacroixd C, Chabrole B, Desguerref I, et al. French Perinatal Cohort Study Group. Persistent mitochondrial dysfunction in HIV-1-exposed but uninfected infants: clinical screening in a large prospective cohort. AIDS 2003, 17: 1769-85.

39.- Hernández S, Morén C, López M, Coll O, Cardellach F, Gratacós E, et al. Perinatal outcomes, mitocondrial toxicity and apoptosis in HIV-treated pregnant women and in-utero-exposed newborn. AIDS 2012; 26: 419-28.

40.- Côté H, Raboud J, Bitnun A, Alimenti A, Money D, Maan E, et al. Perinatal exposure to antiretroviral therapy is associated with increased blood mitochondrial DNA levels and decreased mitochondrial gene expression in infants. J Infect Dis 2008; 198: 851-9. 